\title{
Estudo analítico da influência da correlação entre grandezas no cálculo da incerteza de medição da tensão de resistência mecânica
}

\author{
Analytical study of the correlation influence between \\ quantities in the calculation of the uncertainty in \\ measurement of the mechanical strength tensile
}

José Eduardo Ferreira de Oliveira ${ }^{1}$

\footnotetext{
${ }^{1}$ Laboratório de Metrologia Dimensional - IFPE/Campus Recife. Av. Prof. Luiz Freire, 500, Cidade Universitária - Recife/PE/Brasil CEP 50740-540

e-mail: joseferreira@ recife.ifpe.edu.br
}

\section{RESUMO}

O cálculo da incerteza de medição em ensaios mecânicos é uma etapa primordial para a garantia da confiabilidade dos resultados apresentados. Porém, mesmo atribuindo-se um cálculo estatístico, não necessariamente se obterá um resultado realístico, por não se considerar, muitas vezes, todas as variáveis que contribuem significativamente para a incerteza de medição e por não se considerar o efeito da correlação entre variáveis quando ele se apresenta. Dentro deste contexto, o objetivo deste trabalho é realizar um estudo analítico da influência do coeficiente de correlação no cálculo da incerteza de medição da tensão limite de resistência mecânica, obtido através do ensaio de tração. No estudo experimental, variou-se o número de corpos de prova para a obtenção dos diferentes valores de coeficiente de correlação, com o intuito de se calcular a incerteza de medição considerando-se as grandezas correlacionadas e a incerteza de medição desprezando esse efeito. Através deste estudo foi possível se verificar o quanto a correlação entre a variação da força máxima e a variação do diâmetro da parte reduzida do corpo de prova pode influenciar o resultado da medição, além de apresentar a maneira correta de se calcular a incerteza padronizada combinada considerando a correlação.

Palavras-chave: Coeficiente de correlação, incerteza de medição, ensaio de tração.

\section{ABSTRACT}

The calculation of the uncertainty in measurement in mechanical testing is a primary step to ensuring the reliability of the results. But even assigning a statistical calculation, not necessarily get a realistic result, as often do not consider all variables that contribute significantly to the uncertainty in measurement and does not consider the effect of the correlation between variables. In this context, the aim of this work is perform an analytical study of the correlation coefficient influence in determining of the uncertainty in mea surement of the mechanical strength tensile, obtained by tensile tests. In experimental study varied the number of test specimens to obtain the different values of the correlation coefficient in order to calculate the uncertainty in measurement considering the correlated quantities or not. Through this study it was possible to verify how the correlation between the maximum force and the diameter can influence the measurement result and to present the correct way to calculate the combined uncertainty considering the correlation.

Keywords: Correlation coefficient, uncertainty in measurement, tensile test. 


\section{INTRODUÇÃO}

As grandezas podem ser classificadas de acordo com dois tipos de medição: as diretas e as indiretas. As medições diretas são aquelas em que o resultado da medição é obtido pela aplicação direta do instrumento ou sistema de medição sobre o mensurando. Como exemplo, pode-se citar a medição de um comprimento utilizando-se uma trena. Por sua vez, existem mensurandos cujos resultados de medição são obtidos através de uma expressão matemática envolvendo mais de uma grandeza. Nesse caso, as medições são chamadas de indiretas. Como exemplos, pode-se citar a determinação da tensão limite de resistência mecânica, através do ensaio de tração ou a determinação da dureza Brinell.

Em função da grande quantidade de variáveis de influência sobre uma medição, é fundamental se estabelecer um intervalo, em torno do resultado da medição, que possa abranger, com uma probabilidade específica, os valores que podem ser atribuídos ao mensurando. Este intervalo é chamado de incerteza de medição e é definido como o parâmetro não negativo que caracteriza a dispersão dos valores atribuídos a um mensurando, com base nas informações utilizadas [1]. Segundo WANG [2] , a incerteza é usada como uma ferramenta econômica para estabelecer a alocação ótima de recursos entre a especificação, a fabricação e a verificação.

A incerteza de medição é um valor que se origina da combinação de vários componentes que podem ser estimados com base na distribuição estatística dos resultados de séries de medições, caracterizadas pelo desvio padrão experimental (incerteza tipo A) e através de distribuições de probabilidades consideradas, com base em informações e na experiência adquirida (incerteza tipo B). Logo, o número de fontes de incerteza de medição dependerá do conhecimento e da experiência adquirida no processo de medição. A metodologia para a expressão da incerteza de medição padronizada pelo ISO GUM [3] é apresentada de acordo com a seguinte sequência [4]:

- Levantamento das fontes de incerteza de medição;

- Atribuição da distribuição probabilística para cada fonte de incerteza de medição;

- Determinação dos coeficientes de sensibilidade;

- Determinação da incerteza padronizada combinada;

- Determinação do número de graus de liberdade efetivos;

- Determinação do fator de abrangência e

- Determinação da incerteza expandida de medição.

Inicialmente são levantadas as fontes individuais de incerteza de medição e atribuída uma distribuição probabilística a cada uma delas. Em seguida, as mesmas são combinadas para a obtenção de uma única incerteza com aproximadamente $68 \%$ de confiabilidade (incerteza padronizada combinada) para ser expandida, através do fator de abrangência, obtendo-se assim, uma incerteza de medição para uma confiabilidade de aproximadamente 95\% (incerteza expandida de medição). O ISO GUM [3] provê as bases para o uso das medições e suas incertezas [5]. Ele recomenda o uso de uma incerteza padronizada combinada para expressar a incerteza de medição [్] .

Duas grandezas são consideradas não correlacionadas ou estatisticamente independentes quando as variações aleatórias de uma delas não influenciam as variações aleatórias da outra. Caso contrário elas são ditas correlacionadas ou estatisticamente dependentes. Por sua vez, na determinação da incerteza padronizada combinada em medições indiretas, há a necessidade de se verificar se as grandezas são ou não correlacionadas, pois dependendo do grau de correlação, há uma influência expressiva no valor da incerteza de medição. Este assunto é o tema principal deste trabalho.

\section{REVISÃO BIBLIOGRÁFICA}

\subsection{Determinação da incerteza padronizada combinada para grandezas não correlacionadas}


Cada uma das fontes individuais de incerteza de medição apresenta uma distribuição que lhe é característica. Logo, como o próprio nome já diz, a incerteza padronizada combinada é a incerteza resultante da combinação dessas fontes individuais, chamadas de incertezas padrão. Tais parcelas de contribuição de incerteza de cada uma dessas fontes são dadas através de suas variâncias e covariâncias.

A possibilidade dessa combinação pode ser explicada pelo teorema do limite central, através da Eq.(1).

$W=c_{1} y_{1}+c_{2} y_{2}+\cdots+c_{n} y_{n}$

Onde:

$W=$ grandeza resultante;

$c_{n}=$ coeficiente de sensibilidade associado à n-ésima grandeza componente $\mathrm{e}$

$y_{n}=$ n-ésima grandeza componente com sua respectiva distribuição de probabilidade.

Se todos os $y_{i} \operatorname{com} i$ variando de 1 até $n$ são caracterizados por distribuições normais, então, a distribuição resultante $W$ também será normal. No entanto, mesmo que as distribuições de $y_{1}, y_{2}, \ldots, y_{n}$ não sejam normais, a distribuição resultante $W$ tenderá a se aproximar de uma normal se os termos $y_{1}, y_{2}, \ldots, y_{n}$ forem independentes uns dos outros e a variância da distribuição resultante $W$ for muito maior que qualquer componente obtido pelo produto $\left(\partial f / \partial y_{i}\right)^{2} \times S^{2}\left(y_{i}\right)$, onde $f$ é a função que descreve a grandeza medida, $S^{2}\left(y_{i}\right)$ é a variância de $y_{i}$ e a distribuição associada a $y_{i}$ sendo não normal. Logo, a incerteza padronizada combinada, com aproximadamente $68 \%$ de confiabilidade, é expressa pela Eq.(2).

$u_{c}(y)=\sqrt{\sum_{i=1}^{n}\left(\frac{\partial f}{\partial y_{i}}\right)^{2} \times u^{2}\left(y_{i}\right)}$

Onde:

$u_{c}(y)=$ incerteza padronizada combinada;

$u\left(y_{i}\right)=$ i-ésima fonte de incerteza padrão e

$n=$ número de fontes de incerteza padrão.

A fração $\partial f / \partial y_{i}$ representada por $c_{n}$, mostra o quanto a saída $f$, que representa uma dada função a ser estudada, é influenciada pelo fator de entrada $y_{i}$.

A Eq.(2) é baseada em uma aproximação de primeira ordem da série de Taylor de $Y=f\left(y_{1}, y_{2}, y_{3}, \ldots\right.$, $y_{n}$ ) e expressa o que é denominado no ISO GUM [3] como a lei de propagação de incerteza. É importante salientar que quando há uma expressiva não linearidade da função $f$, há a necessidade de se incluir termos de ordem superior na expansão da série de Taylor para expressar $u_{c}(y)$.

\subsection{Determinação da incerteza padronizada combinada para grandezas correlacionadas}

A Eq.(2) só é válida se todas as grandezas de entrada $y_{i} \operatorname{com} i$ variando de 1 a $n$ forem independentes ou não correlacionadas. Se pelo menos um valor de $y_{i}$ apresentar correlação, a mesma deverá ser considerada na determinação da incerteza padronizada combinada. Nesse caso, a incerteza padronizada combinada é determinada através da Eq.(3) [3].

$u_{c}(y)=\sqrt{\sum_{i=1}^{n}\left(\frac{\partial f}{\partial y_{i}}\right)^{2} \times u^{2}\left(y_{i}\right)+2 \times \sum_{i=1}^{n-1} \sum_{j=i+1}^{n} \frac{\partial f}{\partial y_{i}} \times \frac{\partial f}{\partial y_{j}} \times u\left(y_{i}, y_{j}\right)}$ 
Onde:

$u\left(y_{i}, y_{j}\right)=u\left(y_{j}, y_{i}\right)=$ covariância estimada, associada às grandezas de entrada $y_{i}$ e $y_{\mathrm{j}}$.

Comparando-se as equações 2 e 3, percebe-se um termo adicional na Eq.(3), o qual reflete a influência da correlação entre as grandezas, trabalhadas duas a duas. Se duas variáveis $y_{i}$ e $y_{j}$ são descritas por uma função densidade de probabilidade conjunta $f\left(y_{i}, y_{j}\right)$, então, a covariância de $y_{i}$ e $y_{j}$ é dada pela Eq.(4).

$\operatorname{cov}\left(y_{i}, y_{j}\right)=\int_{-\infty}^{+\infty} \partial y_{i} \int_{-\infty}^{+\infty} y_{i} \cdot y_{j} f\left(y_{i}, y_{j}\right) \partial y_{j}$

O valor da covariância $\operatorname{cov}\left(y_{i}, y_{j}\right)$ ou $v\left(y_{i}, y_{j}\right)$ pode ser estimada por $u\left(y_{i}, y_{j}\right)$, obtida a partir de $n$ valores independentes de observações tanto de $\mathrm{y}_{\mathrm{i}}$ quanto de $\mathrm{y}_{\mathrm{j}}$. $\mathrm{O}$ valor de $u\left(y_{i}, y_{j}\right)$ é obtido a partir da Eq.(5).

$$
u(x, z)=\frac{1}{n \times(n-1)} \times \sum_{i=1}^{n}\left(x_{i}-\bar{X}\right) \times\left(z_{i}-\bar{Z}\right)
$$

Onde:

$\mathrm{x}=$ representação da variável de entrada $y_{i}$;

$\mathrm{z}=$ representação da variável de entrada $y_{j}$;

$\underline{\bar{X}}=$ média aritmética dos valores $x_{i} \mathrm{e}$

$\bar{Z}$ = média aritmética dos valores $z_{i}$.

Para se determinar o grau de correlação entre $y_{i}$ e $y_{j}$, utiliza-se o chamado coeficiente de correlação, de acordo com a Eq.(6), o qual mede a dependência mútua entre essas duas variáveis.

$$
r\left(y_{i}, y_{j}\right)=r\left(y_{j}, y_{i}\right)=\frac{u\left(y_{i}, y_{j}\right)}{\sqrt{u\left(y_{i}, y_{i}\right) \times u\left(y_{j}, y_{j}\right)}}
$$

A Eq.(6) pode ser reescrita da seguinte forma:

$$
r\left(y_{i}, y_{j}\right)=\frac{u\left(y_{i}, y_{j}\right)}{u\left(y_{i}\right) \times u\left(y_{j}\right)}
$$

Onde:

$u\left(y_{i}\right)=$ incerteza tipo A das observações $y_{i} \mathrm{e}$

$u\left(y_{j}\right)=$ incerteza tipo A das observações $y_{j}$

$\mathrm{O}$ valor de $r\left(y_{i}, y_{j}\right)$ encontra-se entre -1 e +1 . Caso as variáveis aleatórias $y_{i}$ e $y_{j}$ sejam independentes, o coeficiente de correlação, bem como sua variância, são iguais a zero. Em resumo, tem-se:

$-1 \leq r\left(y_{i}, y_{j}\right) \leq+1$ (dependência entre as variáveis aleatórias $y_{i}$ e $y_{j}$ ) e

$r\left(y_{i}, y_{j}\right)=0$ (independência entre as variáveis aleatórias $y_{i}$ e $y_{j}$ )

Desta forma, substituindo-se o valor de $u\left(y_{i}, y_{j}\right)$ da Eq. (7) na Eq.(3), obtém-se:

$$
u_{c}(y)=\sqrt{\sum_{i=1}^{n}\left(\frac{\partial f}{\partial y_{i}}\right)^{2} \times u^{2}\left(y_{i}\right)+2 \times \sum_{i=1}^{n-1} \sum_{j=i+1}^{n} \frac{\partial f}{\partial y_{i}} \times \frac{\partial f}{\partial y_{j}} \times u\left(y_{i}\right) \times u\left(y_{j}\right) \times r\left(y_{i}, y_{j}\right)}
$$


A seguir, será apresentado um estudo destacando a influência do número de medições no coeficiente de correlação e, conseqüentemente, na incerteza expandida para o caso da determinação da tensão limite de resistência mecânica, obtida por meio de um ensaio de tração. Este estudo também apresentará a forma como expressar analiticamente a incerteza expandida de medição, bem como os impactos de se desprezar a influência da correlação entre a carga aplicada e o diâmetro medido no comprimento paralelo []] referente a este ensaio.

\section{MATERIAIS E MÉTODOS}

A máquina de tração possui as seguintes características:

- Faixa nominal $=0-10000 \mathrm{kgf}$;

- Menor divisão = 1 kgf;

- Indicador digital;

- Incerteza expandida de medição $=3,5 \mathrm{~N}$ para um fator de abrangência $k_{t}$ de 2,0 (ambos obtidos do certificado de calibração da máquina).

O paquímetro utilizado para a medição dos diâmetros do comprimento paralelo de cada um dos corpos de prova possui as seguintes características:

- Faixa nominal $=0-150 \mathrm{~mm}$;

- Menor divisão $=0,05 \mathrm{~mm}$;

- Incerteza expandida de medição $=0,03 \mathrm{~mm}$ para um fator de abrangência $k_{d}$ de 2,1 (ambos obtidos do certificado de calibração do paquímetro).

A tensão limite de resistência mecânica $\sigma$ é obtida, de acordo com a seguinte equação:

$$
\sigma=\frac{4 \times F}{\pi \times d^{2}}
$$

Onde:

$\sigma=$ tensão limite de resistência mecânica em MPa;

$F=$ força axial em $\mathrm{N} \mathrm{e}$

$d=$ diâmetro do comprimento paralelo em $\mathrm{mm}$.

Este estudo considerou o aço ABNT 4340, realizando o ensaio para cinco corpos de prova, conforme apresentado na Tabela 1.

Tabela 1: Valores da medição da força máxima, juntamente com o seu respectivo diâmetro do comprimento paralelo para cada corpo de prova do aço ABNT 4340

\begin{tabular}{c|c}
\hline Força (N) & Diâmetro (mm) \\
\hline 41510 & 6,00 \\
\hline 42716 & 6,00 \\
\hline 42864 & 6,05 \\
\hline 43026 & 6,05 \\
\hline 43040 & 6,20 \\
\hline
\end{tabular}


Para a determinação da incerteza expandida de medição foram consideradas as seguintes fontes de incerteza padrão:

- Incerteza estatística da força aplicada (tipo A);

- Incerteza herdada da máquina de tração (tipo B);

- Incerteza devida à resolução da máquina de tração (tipo B);

- Incerteza estatística devida à medição dos diâmetros e

- Incerteza herdada do paquímetro.

\section{RESULTADOS}

Os sub-tópicos 4.1 a 4.9 apresentam a sequência para obtenção da incerteza expandida de medição, de acordo com o ISO GUM []], para o aço em questão, cujos valores estão na Tabela 1.

\subsection{Incerteza estatística da força aplicada}

Esta fonte de incerteza padrão, ou seja, com aproximadamente $68 \%$ de confiabilidade é obtida conforme apresentado na Eq.(10).

$$
i_{a}=\frac{S\left(y_{i}\right)}{\sqrt{n}}
$$

Onde:

$i_{a}=$ incerteza estatística da força em $\mathrm{N}$ e

$S\left(y_{i}\right)=$ desvio padrão referente aos $n$ valores de força medidos em $\mathrm{N}$.

A unidade desta fonte de incerteza padrão está em N. Logo, como se pretende obter o valor da incerteza da tensão, deve-se calcular o coeficiente da sensibilidade, derivando-se a Eq.(9) em função da força $F$, obtendo-se a Eq.(11).

$$
c_{1}=\frac{\partial \sigma}{\partial F}=\frac{4}{\pi \times d^{2}}
$$

Onde:

$c_{1}=$ coeficiente de sensibilidade da força em $\mathrm{mm}^{-2}$;

$d$ = diâmetro médio, obtido das $n$ medições do diâmetro do comprimento paralelo em mm.

Considerando-se as forças da Tabela 1, o desvio padrão é igual a 640,60N. Aplicando-se a Eq.(10), tem-se o valor de $i_{a}$ igual a $286,48 \mathrm{~N}$. O coeficiente de sensibilidade $c_{l}$ é obtido da Eq.(11), considerando-se o diâmetro médio obtido dos cinco valores de diâmetro da Tabela 1 , sendo igual a $0,03 \mathrm{~mm}^{-2}$.

\subsection{Incerteza herdada da máquina de tração}

A incerteza expandida da máquina de tração, obtida diretamente do certificado de calibração é uma fonte de incerteza com confiabilidade de aproximadamente 95\%. A fim de se obter a incerteza padrão, deve-se dividir este valor pelo correspondente fator de abrangência, conforme Eq.(12).

$i_{h t}=\frac{U_{t}}{k_{t}}$

Onde: 
$i_{h t}=$ incerteza herdada da máquina de tração em $\mathrm{N}$;

$U_{t}=$ incerteza expandida da máquina de tração que para o estudo em questão é igual a 3,50N e

$k_{t}=$ fator de abrangência associado à incerteza $U_{t}$ e neste caso específico é igual a 2,0.

Como esta é uma fonte de incerteza de força, o coeficiente de sensibilidade $c_{2}$ também é obtido derivando-se a Eq.(9) em função da força $F$, sendo igual ao coeficiente $c_{l}$, conforme Eq.(11). Para o exemplo em questão, $i_{h t}$ é igual a $1,75 \mathrm{~N}$.

\subsection{Incerteza devida à resolução da máquina de tração}

A menor divisão da máquina de ensaio com indicador digital vale $1 \mathrm{kgf}$, o que corresponde a aproximadamente 9,81N. Admitindo-se uma distribuição probabilística retangular, esta fonte de incerteza (tipo B) é quantificada através da Eq. (13).

$$
i_{r t}=\frac{r}{2 \times \sqrt{3}}
$$

Onde:

$i_{r t}=$ incerteza padrão relacionada à resolução da máquina de tração em $\mathrm{N} \mathrm{e}$ $r=$ menor divisão da máquina de tração em $\mathrm{N}$.

Também com relação a esta fonte de incerteza padrão, o coeficiente de sensibilidade $c_{3}$ é igual aos coeficientes $c_{1}$ e $c_{2}$, obtidos da derivada da tensão em função da força. $\mathrm{O}$ valor de $i_{r t}$ é igual a 2,83N.

\subsection{Incerteza estatística devida à medição dos diâmetros}

Esta fonte de incerteza estatística (tipo A) é dada pela Eq.(14).

$$
i_{d}=\frac{S\left(y_{j}\right)}{\sqrt{n}}
$$

Onde:

$i_{d}=$ incerteza estatística da medição dos diâmetros em mm e

$S\left(y_{j}\right)=$ desvio padrão referente aos $n$ valores de diâmetro medidos em mm.

A unidade desta fonte de incerteza padrão está em $\mathrm{mm}$. Logo, como se pretende obter o valor da incerteza da tensão em MPa, deve-se calcular o coeficiente da sensibilidade $c_{4}$, derivando-se a Eq.(9) em função do diâmetro $d$, obtendo-se a Eq.(15).

$$
c_{4}=\frac{\partial \sigma_{R}}{\partial d}=-\frac{8}{\pi} \times \bar{F} \times \frac{1}{\bar{d}^{3}}
$$

Onde:

$c_{4}=$ coeficiente de sensibilidade em N/mm

$\bar{F}=$ força média das $n$ medições realizadas em $\mathrm{Ne}$

$\bar{d}=$ diâmetro médio das $n$ medições realizadas em $\mathrm{mm}$. 
O valor de $i_{d}$ para os dados da Tabela 1 é igual a $0,04 \mathrm{~mm}$. Já o cálculo do coeficiente de sensibilidade $c_{4}$ é dado pela Eq.(15), sendo igual a $-487,81 \mathrm{~N} . \mathrm{mm}^{-3}$. O sinal do coeficiente de sensibilidade deve ser levado em consideração no caso de grandezas de entrada correlacionadas [7]].

\subsection{Incerteza herdada do paquímetro}

Esta fonte de incerteza padrão $i_{h \mathrm{p}}$ é obtida dividindo-se a incerteza expandida $U_{p}$, obtida diretamente do certificado de calibração do paquímetro pelo seu respectivo fator de abrangência $k_{d}$, também encontrado no certificado de calibração do paquímetro. A Eq.(16) apresenta a expressão matemática para o cálculo desta fonte de incerteza.

$$
i_{h p}=\frac{U_{p}}{k_{d}}
$$

Como o paquímetro utilizado nas medições possui incerteza expandida de medição igual a $0,03 \mathrm{~mm}$ para um $k_{d}$ igual a $2,1, i_{h p}$ é igual a $0,01 \mathrm{~mm}$. O coeficiente $c_{5}$ é igual a $c_{4}$.

\subsection{Determinação da incerteza padronizada combinada}

Antes de se determinar a incerteza padronizada combinada, deve-se verificar se há correlação entre a força máxima e o diâmetro do comprimento paralelo. A fim de simplificar a aplicação das equações relacionadas a este cálculo, a Tabela 2 relaciona as variáveis aleatórias gerais com as deste problema específico.

Tabela 2: Atribuição das variáveis gerais das equações com as variáveis específicas do problema

\begin{tabular}{c|c}
\hline Variáveis gerais & Variáveis específicas \\
\hline$y_{1}$ & $F$ \\
\hline$y_{2}$ & $d$ \\
\hline$y_{3}$ & - \\
\hline$y_{4}$ & - \\
\hline$y_{5}$ & - \\
\hline
\end{tabular}

Para os dados da Tabela 1, a força média $\bar{F}$ vale 42631,29 N, o desvio padrão da força $s(F)$ é igual a $640,60 \mathrm{~N}$, o diâmetro médio do comprimento paralelo $\bar{d}$ é igual a $6,06 \mathrm{~mm}$ e o desvio padrão do diâmetro $s(d)$ vale $0,08 \mathrm{~mm}$.

A existência ou não de correlação é verificada através da determinação do coeficiente de correlação, conforme Eq. (7). Neste caso, deve-se, inicialmente, calcular a estimativa da covariância entre $F$ e $d, u(F, d)$, de acordo com a Eq.(5). Neste caso, tem-se: $F_{1}=41510 ; F_{2}=42716 ; F_{3}=42864 ; F_{4}=43026 ; F_{5}=43040 ; d_{1}$ $=6,00 ; d_{2}=6,00 ; d_{3}=6,05 ; d_{4}=6,05$ e $d_{5}=6,20$. Aplicando-se a Eq. (5) para estes dados, obtém-se:

$$
\begin{aligned}
& u(F, d)=\frac{1}{5 \times(5-1)} \times[(41510-42631,29) \times(6,00-6,06)+(42716-42631,29) \times(6,00-6,06)+ \\
& +(42864-42631,29) \times(6,05-6,06)+(43026-42631,29) \times(6,05-6,06)+(43040-42631,29) \times \\
& \times(6,20-6,06)]=5,66 N . \mathrm{mm}
\end{aligned}
$$

O valor do coeficiente de correlação $r(F, d)$, dado pela Eq.(7) é, portanto:

$$
r(F, d)=\frac{5,66}{286,48 \times 0,04}=0,54
$$


Verifica-se, portanto a existência de correlação direta entre as variáveis F e d. Logo, a incerteza padronizada combinada é calculada de acordo com a Eq.(8) que adaptada a este problema, é apresentada na Eq.(17).

$$
\begin{aligned}
& u_{c}(y)=\left\{\left(c_{1}^{2} \times i_{a}^{2}\right)+\left(c_{2}^{2} \times i_{h t}^{2}\right)+\left(c_{3}^{2} \times i_{r t}^{2}\right)+\left(c_{4}^{2} \times i_{d}^{2}\right)+\left(c_{5}^{2} \times i_{h p}^{2}\right)\right]+2 \times\left[\frac{\partial \sigma}{\partial y_{1}} \times \frac{\partial \sigma}{\partial y_{2}} \times u\left(y_{1}\right) \times u\left(y_{2}\right) \times r\left(y_{1}, y_{2}\right)+\right. \\
& +\frac{\partial \sigma}{\partial y_{1}} \times \frac{\partial \sigma}{\partial y_{3}} \times u\left(y_{1}\right) \times u\left(y_{3}\right) \times r\left(y_{1}, y_{3}\right)+\frac{\partial \sigma}{\partial y_{1}} \times \frac{\partial \sigma}{\partial y_{4}} \times u\left(y_{1}\right) \times u\left(y_{4}\right) \times r\left(y_{1}, y_{4}\right)+\frac{\partial \sigma}{\partial y_{1}} \times \frac{\partial \sigma}{\partial y_{5}} \times u\left(y_{1}\right) \times u\left(y_{5}\right) \times r\left(y_{1}, y_{5}\right)+ \\
& +\frac{\partial \sigma}{\partial y_{2}} \times \frac{\partial \sigma}{\partial y_{3}} \times u\left(y_{2}\right) \times u\left(y_{3}\right) \times r\left(y_{2}, y_{3}\right)+\frac{\partial \sigma}{\partial y_{2}} \times \frac{\partial \sigma}{\partial y_{4}} \times u\left(y_{2}\right) \times u\left(y_{4}\right) \times r\left(y_{2}, y_{4}\right)+\frac{\partial \sigma}{\partial y_{2}} \times \frac{\partial \sigma}{\partial y_{5}} \times u\left(y_{2}\right) \times u\left(y_{5}\right) \times r\left(y_{2}, y_{5}\right)+ \\
& \left.\left.\frac{\partial \sigma}{\partial y_{3}} \times \frac{\partial \sigma}{\partial y_{4}} \times u\left(y_{3}\right) \times u\left(y_{4}\right) \times r\left(y_{3}, y_{4}\right)+\frac{\partial \sigma}{\partial y_{3}} \times \frac{\partial \sigma}{\partial y_{5}} \times u\left(y_{3}\right) \times u\left(y_{5}\right) \times r\left(y_{3}, y_{5}\right)++\frac{\partial \sigma}{\partial y_{4}} \times \frac{\partial \sigma}{\partial y_{5}} \times u\left(y_{4}\right) \times u\left(y_{5}\right) \times r\left(y_{4}, y_{5}\right)\right]\right\}
\end{aligned}
$$

Como as variáveis $y_{3}, y_{4}$, e $y_{5}$ não foram obtidas estatisticamente, os coeficientes de correlação relacionados às mesmas são nulos, obtendo-se, portanto, a Eq.(18). Por sua vez, $u\left(y_{1}\right)=i_{a}$ e $u\left(y_{2}\right)=i_{d}$. A derivada de $\sigma$ em função de $F$ e em função de $d$ são dadas pela Eq.(11) e pela Eq.(15), respectivamente.

$$
u_{c}(y)=\sqrt{\left(c_{1}^{2} \times i_{a}^{2}\right)+\left(c_{2}^{2} \times i_{h t}^{2}\right)+\left(c_{3}^{2} \times i_{r t}^{2}\right)+\left(c_{4}^{2} \times i_{d}^{2}\right)+\left(c_{5}^{2} \times i_{h p}^{2}\right)+2 \times \frac{\partial \sigma}{\partial F} \times \frac{\partial \sigma}{\partial d} \times i_{a} \times i_{d} \times r(F, d)}
$$

Substituindo-se os valores na Eq.(18), obtém-se o valor da incerteza padronizada combinada igual a $16,65 \mathrm{MPa}$.

\subsection{Determinação do número de graus de liberdade efetivos}

O número de graus de liberdade efetivos $v_{\text {eff }}$ é obtido por meio da Eq.(19) de Welch-Satterthwaite. O valor do número de graus de liberdade associado a cada fonte de incerteza dependerá do tipo de incerteza, ou seja, se ela é do tipo A ou do tipo B, como apresentado na Tabela 3. O valor de $v_{\text {eff }}$ é utilizado para a determinação do fator de abrangência $\mathrm{k}$.

$$
v_{e f f}=\frac{u_{c}^{4}(y)}{\sum_{i=1}^{N} \frac{u_{i}^{4}(y)}{v_{i}}}
$$

Onde:

$u_{i}(y)=\mathrm{i}$-ésima fonte de incerteza padrão, multiplicada pelo seu respectivo coeficiente de sensibilidade e $v_{i}=$ graus de liberdade referentes à i-ésima fonte de incerteza padrão.

Tabela 3: Número de graus de liberdade de acordo com cada fonte de incerteza

\begin{tabular}{c|l}
\hline TIPO DE INCERTEZA & \multicolumn{1}{c}{ GRAUS DE LIBERDADE } \\
\hline tipo A & $v=n-1$, onde $n$ é o número de medições realizadas. \\
\hline tipo B & $v=\infty$ \\
\hline
\end{tabular}

Aplicando-se os dados na Eq.(19), obtém-se:

$$
v_{e f f}=\frac{u_{c}(y)^{4}}{\frac{\left(c_{1} \times i_{a}\right)^{4}}{4}+\frac{\left(c_{2} \times i_{h t}\right)^{4}}{\infty}+\frac{\left(c_{3} \times i_{r t}\right)^{4}}{\infty}+\frac{\left(c_{4} \times i_{d}\right)^{4}}{4}+\frac{\left(c_{5} \times i_{h p}\right)^{4}}{\infty}}
$$


Substituindo-se os valores na Eq. (20), o valor de $v_{\text {eff }}$ encontrado é igual a 2,72.

\subsection{Determinação do fator de abrangência}

O fator de abrangência $k$, por sua vez, é um fator numérico utilizado como multiplicador da incerteza padronizada combinada para obter uma incerteza expandida [9]. Esse fator é determinado de acordo com a confiabilidade requerida. $\mathrm{Na}$ grande maioria dos casos, ele situa-se entre 2 e 3 . Como em medições dimensionais o espaço amostral é pequeno, ou seja, são realizadas poucas medições para uma mesma dimensão, a curva que melhor descreve tal situação não é uma distribuição normal, mas sim uma distribuição t-Student, cuja função de densidade de probabilidade é apresentada na Eq.(21) [10]. A Tabela 4 apresenta o percentual do nível de confiança para diferentes valores de $k$, e a função gama $\Gamma$ é dada pela Eq.(22). Para o valor de $v_{\text {eff }}$ de 2,72, $k$ vale 4,53 .

$$
f(x)=\frac{\Gamma\left(\frac{n+1}{2}\right)}{\sqrt{n \cdot \pi} \cdot \Gamma\left(\frac{n}{2}\right)} \times \frac{1}{\left(1+\frac{z^{2}}{n}\right)^{\frac{n+1}{2}}}
$$

$\Gamma(n+1)=n !$

Tabela 4: Percentual do nível de confiança para diferentes valores de k, considerando-se a distribuição t-Student

\begin{tabular}{c|c}
\hline $\mathbf{k}$ & NíVEL DE CONFIANÇA (\%) \\
\hline 1 & 68,27 \\
\hline 2 & 95,45 \\
\hline 3 & 99,73 \\
\hline
\end{tabular}

À medida que se aumenta o número de medições, a distribuição t-Student tenderá a uma normal. Para um tamanho de amostra menor que 120 , o desvio padrão amostral " $S$ " se torna uma melhor estimativa do desvio padrão da população [11].

\subsection{Determinação da incerteza expandida de medição}

A incerteza expandida de medição $U$ com $95,45 \%$ de confiabilidade é obtida, multiplicando-se a incerteza padronizada combinada pelo fator de abrangência, conforme Eq.(23).

$U=k \times u_{c}$

A determinação da incerteza de medição acima descrita foi aplicada para os dados da Tabela 1, considerando, para cada um deles, a utilização de 3, 4 e 5 corpos de prova. As Tabelas de 5, 6 e 7 apresentam os valores da incerteza de medição, considerando-se o efeito da correlação e desprezando-se este efeito, com o intuito de se comparar tais valores com o número de corpos de prova utilizados, bem como com o coeficiente de correlação. $\mathrm{O}$ coeficiente $\alpha$ expressa a razão entre a incerteza expandida considerando-se o efeito da correlação e a incerteza expandida desprezando-se a correlação. Por sua vez, a variável $b$ expressa a diferença entre os valores das incertezas considerando-se a correlação e a desprezando-a. 
Tabela 5: Valores do aço ABNT 4340, considerando-se cinco corpos de prova

\begin{tabular}{c|c|c|c|c|c|c}
\hline $\begin{array}{c}\text { № DE CORPOS DE } \\
\text { PROVA }\end{array}$ & $\begin{array}{c}\text { MÉDIA DA } \\
\text { TENSÃO } \\
(\mathrm{MPa})\end{array}$ & $\boldsymbol{r}\left(\boldsymbol{x}_{i}, \boldsymbol{x}_{j}\right)$ & $\begin{array}{c}\text { U (MPa) COM } \\
\text { CORRELAÇÃO }\end{array}$ & $\begin{array}{c}\text { U (MPa) SEM } \\
\text { CORRELAÇÃO }\end{array}$ & $\boldsymbol{a}$ & $\begin{array}{c}\boldsymbol{b} \\
(\mathrm{MPa})\end{array}$ \\
\hline 5 & 1478 & 0,5374 & 75,35 & 52,57 & 1,43 & 22,78 \\
\hline
\end{tabular}

Tabela 6: Valores do aço ABNT 4340, considerando-se quatro corpos de prova

\begin{tabular}{c|c|c|c|c|c|c}
\hline $\begin{array}{c}\text { № DE CORPOS DE } \\
\text { PROVA }\end{array}$ & $\begin{array}{c}\text { MÉDIA DA } \\
\text { TENSÃO } \\
(\mathrm{MPa})\end{array}$ & $\boldsymbol{r}\left(\boldsymbol{x}_{i}, \boldsymbol{x}_{j}\right)$ & $\begin{array}{c}\mathbf{U}(\mathrm{MPa}) \text { COM } \\
\text { CORRELAÇÃO }\end{array}$ & $\begin{array}{c}\mathbf{U}(\mathrm{MPa}) \text { SEM } \\
\text { CORRELAÇÃo }\end{array}$ & $\boldsymbol{a}$ & $\begin{array}{c}\boldsymbol{b} \\
(\mathrm{MPa})\end{array}$ \\
\hline 4 & 1492 & 0,6951 & 51,13 & 38,25 & 1,34 & 12,88 \\
\hline
\end{tabular}

Tabela 7: Valores do aço ABNT 4340, considerando-se três corpos de prova

\begin{tabular}{c|c|c|c|c|c|c}
\hline $\begin{array}{c}\text { № DE CORPOS DE } \\
\text { PROVA }\end{array}$ & $\begin{array}{c}\text { MÉDIA DA } \\
\text { TENSÃO } \\
(\mathrm{MPa})\end{array}$ & $\boldsymbol{r}\left(\boldsymbol{x}_{\boldsymbol{i}}, \boldsymbol{x}_{j}\right)$ & $\begin{array}{c}\mathbf{U}(\mathrm{MPa}) \text { COM } \\
\text { CORRELAÇÃO }\end{array}$ & $\begin{array}{c}\mathbf{U}(\mathrm{MPa}) \text { SEM } \\
\text { CORRELAÇÃO }\end{array}$ & $\boldsymbol{a}$ & $\begin{array}{c}\boldsymbol{b} \\
(\mathrm{MPa})\end{array}$ \\
\hline 3 & 1490 & 0,5838 & 197,69 & 53,35 & 3,71 & 144,34 \\
\hline
\end{tabular}

\section{DISCUSSÃO}

Em todos os casos estudados, constata-se a existência de correlação entre a força aplicada e o diâmetro do comprimento paralelo, uma vez que $r\left(x_{i}, x_{j}\right)$ é diferente de zero e se encontra entre $-1 \mathrm{e}+1$. Por outro lado, em todos os casos a correlação foi direta, pois $r\left(x_{i}, x_{j}\right)$ se apresentou sempre maior que zero.

A maior diferença " $b$ " foi verificada na utilização de apenas três corpos de prova, conforme Tabela 7. Para essa situação, não se verifica o maior coeficiente de correlação. A explicação para essa enorme diferença de 144,34MPa para a variável " $b$ ” se deveu à diferença nos fatores de abrangência para a incerteza de medição considerando a correlação e a incerteza desconsiderando-a. Para a incerteza expandida desconsiderando-se o efeito da correlação, o valor de k vale 2,87, ao passo que para a incerteza com correlação, este valor aumentou para 13,97, ou seja, quase 5 vezes maior. Essa maior diferença " $b$ ” corresponde a 9,69\% da tensão limite de resistência mecânica média (1490MPa), o que corrobora para a necessidade de se apresentar uma incerteza de medição realística, levando-se em consideração o efeito da correlação na sua determinação.

Para todos os três casos estudados, a incerteza expandida considerando-se a correlação foi maior que a incerteza sem o efeito da mesma, o que implica, conseqüentemente, em valores de $\alpha$ superiores a 1.

\section{CONCLUSÕES}

Através da análise da diferença apresentada na incerteza de medição considerando-se a correlação e desprezando-a para o caso de se utilizar apenas três corpos de prova, em função do aumento no fator de abrangência “ $k$ ”, constata-se um resultado da incerteza não realístico, o que se leva a concluir que a utilização de apenas três corpos de prova para este ensaio é insuficiente. Considerando-se quatro ou cinco corpos de prova, obtiveram-se valores realísticos da incerteza de medição e, conseqüentemente, do resultado do ensaio.

A adoção de uma completa sistemática para a correta expressão da incerteza de medição representa uma ação que implica, diretamente, na garantia da qualidade do resultado apresentado. Considerando-se o exemplo 
apresentado, verifica-se a importância fundamental de se expressar a incerteza de medição de acordo com a sistemática apresentada pelo ISO GUM []] , de modo que o valor médio da tensão limite de resistência mecânica mais ou menos a incerteza expandida de medição fique dentro dos limites de tolerância do referido material e que o valor dessa incerteza apresente uma confiabilidade de pelo menos $95 \%$.

\section{BIBLIOGRAFIA}

[1] VIM - Vocabulário Internacional de Metrologia: conceitos fundamentais e gerais e termos associados. Duque de Caxias, RJ: INMETRO, 2012.

[2] WANG, J. X., JIANG, X., MA, L. M., et al., Decision Rules for Workpieces based on Total Uncertainty, Springer Verlag London Limited, London, 2005.

[3] GUM - Avaliação de dados de medição: guia para a expressão de incerteza de medição. Duque de Caxias, RJ: INMETRO/CICMA/SEPIN, 2012

[4] OLIVEIRA, J. E. F. de. Desenvolvimento de um programa computacional para a integração de dados de projeto, fabricação e medição de peças torneadas com ênfase na síntese de tolerâncias dimensionais determinísticas, Tese D.Sc., Universidade Federal de Pernambuco, Recife, 2009.

[5] SLEELE, A. "Analysis of measurement comparisons". In: Imeko World Congress, XVIII. Metrology for a sustainable development. Rio de Janeiro, Brazil, 2006.

[6] WOOD, B. M., DOUGLAS, R. J. "Confidence-interval interpretation of a measurement pair for quantifying a comparison", Metrologia, v.35, pp. 187-196, Sèvres, 1998.

[7] EA-4/02. Expressão da incerteza de medição na calibração. Primeira edição brasileira em língua portuguesa do EA-4/02 - Rio de Janeiro: INMETRO, ABNT, SBM, 1999. ISBN 85-86768-04-9.

[8] ABNT NBR ISO 6892-1. Materiais metálicos - Ensaio de tração. Parte 1: Método de ensaio à temperatura ambiente. ABNT, Rio de Janeiro, 2013.

[9] UNE-EN ISO 14253-1. Especificación geométrica de productos (GPS). Parte 1: Reglas de decisión para probar la conformidad o no conformidad com las especificaciones. Norma española, Génova, 1998.

[10] KREYSZIG, E. Statistische Methoden und Ihre Anwendungen, Vandenhoeck \& Ruprecht, Göttingen, 1985.

[11] LEVINE, D., BERENSON, M., STEPHAN, D., Estatística: teoria e aplicações, Editora LTC, Rio de Janeiro, 2000. 